\title{
Clinical evaluation of endovascular repair of abdominal aortic aneurysm based on long-term experiences
}

\author{
Piotr Kulig ${ }^{1}$, Krzysztof Lewandowski $^{1}$, Bogusław Rudel ${ }^{2}$, Maciej Chwała ${ }^{1}$, Marek Piwowarczyk ${ }^{3}$, Wojciech Mrowiecki ${ }^{3}$ \\ ${ }^{1}$ Department of Vascular Surgery and Angiology, Brothers of Mercy St. John of God Hospital, Krakow, Poland \\ ${ }^{2}$ Department of Internal Medicine and Angiology, Brothers of Mercy St. John of God Hospital, Krakow, Poland \\ ${ }^{3}$ Department of Vascular Surgery, University Hospital, Krakow, Poland
}

Videosurgery Miniinv DOI: https://doi.org/10.5114/wiitm.2020.93984

\begin{abstract}
Introduction: The endovascular method as a less invasive treatment for patients with abdominal aortic aneurysm $(A A A)$ has become an alternative to conventional open surgery.

Aim: The objective of the present study was to analyse the outcomes of endovascular treatment of AAA patients in long-term observation.

Material and methods: A group of 236 AAA patients subjected to planned endovascular aneurysm repair (EVAR) between 2010 and 2015 was reviewed. Rates of mortality, surgical complications and re-interventions were collected in the separate time periods, i.e. up to 30 days after surgery, 30 days to 3 years, and from 3 to 5 years after surgery. Cumulative rates of these parameters were evaluated in the short-term (up to 30 days after surgery), medium-term (up to 3 years), and long-term (up to 5 years after surgery) perspective.

Results: The median age of patients was 75 years, and the most common comorbidities were arterial hypertension (54\%) and ischaemic heart disease (52\%). Cumulative short-, medium- and long-term mortality rates were $2.5 \%$, $14.2 \%$ and $28.9 \%$, respectively. Total rates of surgical complications in short-, medium- and long-term observation were $7.6 \%, 12.6 \%$ and $17.5 \%$, respectively. The cumulative rate of re-interventions ranged from $4.2 \%$ to $11.4 \%$.

Conclusions: In the consecutive time periods, the increase in the percentage of surgical complications and re-interventions increased gradually, in contrast to mortality, where the curve grew significantly, which is expected due to the aging and numerous comorbidities in the observed group of patients.
\end{abstract}

Key words: abdominal aortic aneurysm, long-term outcomes, endovascular aneurysm repair.

\section{Introduction}

Elective treatment of patients with an abdominal aortic aneurysm (AAA) has undergone a remarkable evolution over the last two decades. The traditional open laparotomy used for surgical repair has been gradually replaced by less invasive interventions, such as the placement of endovascular stents (endovascular aneurysm repair - EVAR). The latter procedure is associated with less traumatic stress and lower pain scores, leading to shorter hospitalization and faster recovery in comparison to open surgery [1-4]. Reports from the United States show that EVAR has become the most frequently used type of intervention for AAA [5-7]. In Poland, the endovascular method is the only alternative to the conventional open surgical treatment of this group of patients.

The endovascular repair of AAA with implanted stent grafts is associated with some inherent draw-

\section{Address for correspondence}

Piotr Kulig, Department of Vascular Surgery and Angiology, Brothers of Mercy St. John of God Hospital, 11 Trynitarska St, 31-252 Krakow, Poland, phone: +48660351 374, e-mail: Kuligos22@interia.pl 
backs. The major complication, both in short- and long-term perspectives, is formation of characteristic leaks. In some cases, especially in the type I leaks, such complications require surgical interventions [8-12]. The cost of equipment required to perform repair using the endovascular technique is higher than the costs of an open procedure. Moreover, the benefits of endovascular treatment of AAA compared to the open surgery are maintained mostly in the short-term period and are comparable in longterm observation [13-15].

\section{Aim}

The aim of the present study was to evaluate time-related changes in morbidity and mortality rates among 236 patients subject to planned EVAR between 2010 and 2015. Cumulative rates of mortality, surgical complications, and re-interventions after endovascular treatment of AAA were compared with world reports.

\section{Material and methods}

Medical records of 236 patients with AAA subject to elective implantation of the stent graft using the endovascular method from 2010 to 2015 in the Department of Vascular Surgery and Angiology, at the Brothers of Mercy St. John of God Hospital in Cracow were reviewed. Computed tomographic (CT) angiography (CTA) of the abdominal aorta was performed in all patients prior to treatment using a 32-row spiral AQUILION 32 scanner (Toshiba). Generally, patients were qualified for intervention based on the AAA diameter above $5-5.5 \mathrm{~cm}$ or the annual growth over $0.5-1.0 \mathrm{~cm}$. Selection of open and endovascular repair was based mainly on the anatomy of AAA, patients' age and their general health condition. Prior to EVAR, all patients were subject to routine biochemical blood analysis and echocardiography, and their risk of surgery-related morbidity and mortality was evaluated with the V-Possum scale.

All repairs were performed in the Endovascular Therapy Unit of the hospital, using dedicated radiological equipment (Philips). Local or subarachnoid anaesthesia was used in the majority of the patients, with a few patients requiring general anaesthesia. Stent grafts were deployed using systems of various manufacturers, including Zenith COOK, Endurant Medtronic, Excluder Gore, Jotec E-tegra, etc.
Follow-up examinations in the Regional Outpatient Centre for Vascular Diseases of the Hospital were generally planned $1,6,12,18,24,36$ and 60 months after the surgery. Additionally, patients were regularly evaluated by phone interviews, and dates of death were verified using data obtained from the census registry office.

Rates of mortality, surgical complications and re-interventions were evaluated in three separate periods following the surgery - up to 30 days after surgery (236 patients), 30 days to 3 years (180 patients), and 3 to 5 years after surgery (102 patients).

Cumulative rates of mortality, surgical complications and re-interventions were assessed in the short-term (up to 30 days after surgery), medium-term (up to 3 years), and long-term (up to 5 years after surgery) perspective.

\section{Statistical analysis}

Statistical analysis was performed using the Statistica 12 software package (StatSoft Polska). Comparison between observed and predicted short-term mortality according to V-Possum was evaluated by the Hosmer-Lemeshow test. Up to the medium- and long-term perspective we used the dynamic series methods for which fixed basis change indexes of mortality, surgical complications and re-interventions were calculated.

\section{Results}

The study population consisted mostly of male elderly patients with numerous co-morbidities (Table I). The median predicted morbidity and mortality risk calculated using the V-Possum score were respectively $27.1 \%$ and $2.9 \%$. The ejection fraction of the left ventricle and basic biochemical parameters of blood, such as haemoglobin, urea and creatinine levels, were within the reference ranges in the majority of the patients. Nicotine addiction was recorded in the medical history of 61 (26\%) patients. The median AAA diameter was 59 mm (IQR 54-65) and clinical manifestations of AAA were recorded in 45 (19\%) patients (Table II). The median duration of surgery was 105 minutes and the median volume of administered Optiray contrasting agent was $110 \mathrm{ml}$.

In the first of the investigated time periods, i.e. up to 30 days after surgery, mortality was $2.5 \%$ and surgical complications occurred in 18 (7.6\%) patients (Table III). Thrombosis and occlusion of the stent graft 
Table I. Demographic and clinical-pathological characteristics of patients

\begin{tabular}{|lc|}
\hline Parameter & Results \\
\hline Number of patients, $n$ & 236 \\
\hline Age [years], (median, IQR) & $75(69-80)$ \\
\hline Male/female, $n$ (\%) & $204(86) / 32(14)$ \\
\hline Comorbidities, $n$ (\%): & $84(36)$ \\
\hline General arteriosclerosis & $127(54)$ \\
\hline Blood hypertension & $122(52)$ \\
\hline Ischaemic heart disease & $17(7)$ \\
\hline Heart valve disorders & $37(16)$ \\
\hline Arrhythmia & $47(19.9)$ \\
\hline Diabetes mellitus & $21(8.9)$ \\
\hline Chronic renal failure & $34(14.4)$ \\
\hline Lung disease & $19(8.0)$ \\
\hline Thyroid disease & $17(7)$ \\
\hline Oncological disease & $61(26)$ \\
\hline Nicotine addiction, $n$ (\%) & $55(43-60)$ \\
\hline Ejection fraction (\%), median (IQR) & $27.1(20.9-33.6)$ \\
\hline V-Possum morbidity (\%), median (IQR) & $13.4(12-14.4)$ \\
\hline V-Possum mortality (\%), median (IQR) & $7.7(6-9.7)$ \\
\hline Haemoglobin [g/dl], median (IQR) & $(79-121.7)$ \\
\hline Urea [mmol/I], median (IQR) & $2.9)$ \\
\hline Creatinine [ $\mu$ mol/I], median (IQR) & \\
\hline
\end{tabular}

with acute ischaemia of the lower limb was reported in $6(2.5 \%)$ patients. Leaks occurred in 6 cases: type I (3 patients) and type II (3 patients). Infections and bleeding at that access site in the groin were identified in $4(1.7 \%)$ and $2(0.8 \%)$ patients, respectively. Ten (4.2\%) patients required re-interventions after endovascular treatment. All patients with thrombosis and occlusion of the stent graft with acute ischaemia of the lower limb required re-interventions. In 5 cases femorofemoral bypass surgery was carried out, and one patient was subjected to thrombectomy of the left and right iliac limbs, with angioplasty and implantation of a stent in the right iliac limb. In all patients with type I leak during short-term observation, re-intervention, which consisted of stent graft reimplantation, was also performed. Patients with type II leak and infection of the wound in the
Table II. Characteristics of abdominal aortic aneurysm (AAA) and perioperative features of the implanted stent graft

\begin{tabular}{|c|c|}
\hline Parameter & Results \\
\hline Number of patients, $n$ & 236 \\
\hline AAA diameter [mm], median (IQR) & $59(54-65)$ \\
\hline Symptomatic AAA yes/no, $n(\%)$ & $45(19) / 191(81)$ \\
\hline \multicolumn{2}{|l|}{$\begin{array}{l}\text { Concomitant aneurysm of iliac arteries, } \\
n(\%):\end{array}$} \\
\hline Right common iliac artery & $58(25)$ \\
\hline Left common iliac artery & $52(22)$ \\
\hline Right external iliac artery & $1(0.4)$ \\
\hline Right internal iliac artery & $7(3)$ \\
\hline Left external iliac artery & $0(0)$ \\
\hline Left internal iliac artery & $14(6)$ \\
\hline $\begin{array}{l}\text { Concomitant thoracic aorta aneurysm, } \\
n(\%)\end{array}$ & $3(1)$ \\
\hline Operation time [min], median (IQR) & $105(89-125)$ \\
\hline \multicolumn{2}{|l|}{ Anaesthesia, $n(\%)$ : } \\
\hline Local & $105(45)$ \\
\hline Subarachnoid & $86(36)$ \\
\hline General & $45(19)$ \\
\hline \multicolumn{2}{|l|}{ Type of implanted stent graft, $n(\%)$ : } \\
\hline Zenith COOK & $69(29)$ \\
\hline Endurant Medtronic & $78(33)$ \\
\hline Excluder Gore & $36(15)$ \\
\hline Jotec E-tegra & $47(20)$ \\
\hline Other & $6(3)$ \\
\hline $\begin{array}{l}\text { Covering of external iliac arteries yes/no, } \\
n(\%)\end{array}$ & $87(37) / 149(63)$ \\
\hline $\begin{array}{l}\text { Volume of administered Optiray } \\
\text { contrasting agent [ml], median (IQR) }\end{array}$ & $110(97-132)$ \\
\hline Hospitalization [days], median (IQR) & $6(4-8)$ \\
\hline
\end{tabular}

groin did not require any surgical intervention. In 1 patient with bleeding from the wound in the groin, a surgical revision of the wound was performed, with haemostasis and haematoma evacuation, while in the case of 1 patient conservative treatment and compression were applied.

The cause of death was myocardial infarction in 3 patients, occlusion of the stent graft with acute 
Table III. Number and rate of mortality, surgical complications and re-interventions in separate periods of time

\begin{tabular}{|c|c|c|c|}
\hline Period of time & $\begin{array}{l}\text { Up to } 30 \text { days } \\
\text { after surgery }\end{array}$ & $\begin{array}{l}30 \text { days to } 3 \text { years } \\
\text { after surgery }\end{array}$ & $\begin{array}{l}3 \text { to } 5 \text { years } \\
\text { after surgery }\end{array}$ \\
\hline Number of patients, $n$ & 236 & 180 & 102 \\
\hline Mortality, $n(\%)$ & $6(2.5)$ & $21(11.7)$ & $15(14.7)$ \\
\hline Surgical complications, $n$ (\%) & $18(7.6)$ & $9(5)$ & $5(4.9)$ \\
\hline Type I leak, $n$ (\%) & $3(1.3)$ & $3(1.7)$ & $3(2.9)$ \\
\hline Type II leak, $n$ (\%) & $3(1.3)$ & $2(1.1)$ & $0(0)$ \\
\hline Type IV leak, n (\%) & $0(0)$ & $0(0)$ & $1(1.0)$ \\
\hline Type V leak, $n$ (\%) & $0(0)$ & $2(1.1)$ & $1(1.0)$ \\
\hline $\begin{array}{l}\text { Thrombosis and stent graft occlusion with } \\
\text { acute ischaemia of a lower limb, } n(\%)\end{array}$ & $6(2.5)$ & $2(1.1)$ & $0(0)$ \\
\hline Bleeding from wound in the groin, $n(\%)$ & $2(0.8)$ & $0(0)$ & $0(0)$ \\
\hline Infection of wound in the groin, $n(\%)$ & $4(1.7)$ & $0(0)$ & $0(0)$ \\
\hline Re-interventions, $n(\%)$ & $10(4.2)$ & $6(3.3)$ & $4(3.9)$ \\
\hline
\end{tabular}

ischaemia of a lower limb in 2 patients and hypovolaemic shock caused by leak type I in 1 patient.

The observed and predicted short-term mortality according to the V-Possum scale was not statistically significantly different, respectively $2.5 \%$ and $2.9 \%$ ( $p=0.7993)$.

In the time period from 30 days to 3 years following surgery (180 patients) the mortality rate was $11.7 \%$ and surgical complications occurred in 9 (5\%) patients. The most frequent complications were

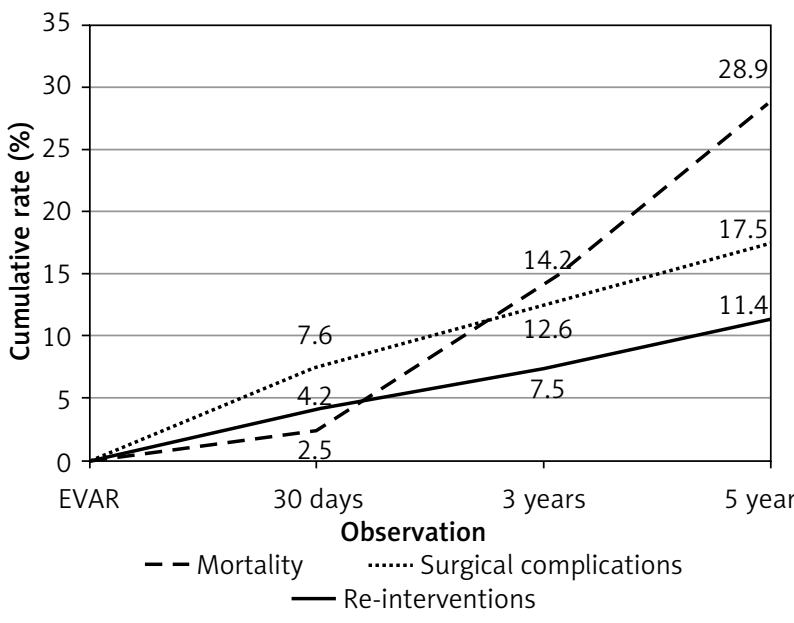

Figure 1. Mortality, surgical complications and re-interventions in short-, middle- and longterm observation - cumulative curves leaks (7, 3.9\%), including type I in 3 cases (1.7\%), type II in 2 (1.1\%) and type $V$ in 2 (1.1\%). In 2 cases $(1.1 \%)$, there was thrombosis and occlusion of the stent graft with acute ischaemia of the lower limb. During this period, 6 patients (3.3\%) had re-intervention due to complications (Table III). Each patient with type I leak and thrombosis and occlusion of the stent graft with acute ischaemia of a lower limb was subject to re-interventions. Patients with type I leak had reimplantation of a stent graft. Thrombosis and occlusion of the stent graft with acute ischaemia of a lower limb was treated with femorofemoral bypass with thrombectomy. In the case of 1 patient with type $\checkmark$ leak, reimplantation of a stent graft was applied.

The main cause of death in patients was comorbidities, especially ischaemic heart disease. In 1 patient, death was caused by occlusion of the stent graft with acute ischaemia of the lower limb.

The mortality rate for the period from 3 to 5 years following the surgery (102 patients) was $14.7 \%$ and surgical complications occurred in 5 (4.9\%) of the patients. All of these complications were leaks, i.e. type I in 3 cases (2.9\%), type IV in 1 (1.0\%) and type V in 1 (1.0\%). Re-intervention in the last time period subject to our research took place in 4 (3.9\%) cases (Table III). In 3 patients with type I leaks and 1 patient with type IV leak, reimplantation of the stent graft was performed. The main cause of death in patients was comorbidities, especially ischaemic heart 
Table IV. Change in dynamics of mortality, surgical complications and re-interventions in the medium- and long-term perspective

\begin{tabular}{|lccc|}
\hline $\begin{array}{l}\text { Analysed parameter } \\
\text { of complications }\end{array}$ & \multicolumn{3}{c|}{ Dynamics index with a fixed basis } \\
\cline { 2 - 4 } & 30 days versus 30 days & 3 years versus 30 days & 5 years versus 30 days \\
\hline Mortality $[\mathrm{m}]$ & $\mathrm{i}_{\mathrm{m}-30 \text { days }}=1$ & $\mathrm{i}_{\mathrm{m}-3 \text { years }}=6.68$ & $\mathrm{i}_{\mathrm{m}-5 \text { years }}=27,4$ \\
\hline Surgical complications $[\mathrm{sc}]$ & $\mathrm{i}_{\mathrm{sc}-30 \text { days }}=1$ & $\mathrm{i}_{\mathrm{sc}-3 \text { years }}=1.66$ & $\mathrm{i}_{\mathrm{sc}-5 \text { years }}=2.3$ \\
\hline Re-interventions [ri] & $\mathrm{i}_{\mathrm{r} \text { - }-30 \text { days }}=1$ & $\mathrm{i}_{\mathrm{r}-3 \text { years }}=1.79$ & $\mathrm{i}_{\mathrm{r}-5 \text { years }}=2.7$ \\
\hline
\end{tabular}

disease. No causal relationship with stent graft implantation was observed.

Figure 1 shows time-related changes in the $\mathrm{cu}$ mulative rates of mortality, surgical complications, and re-interventions. There was a marked increasing trend in mortality from $2.5 \%$ to $14.2 \%$ and finally $28.9 \%$. Total rates of surgical complications and re-interventions rose more gradually.

Change in dynamics of mortality, surgical complications and re-interventions in the medium- and long-term perspective are presented in Table IV.

Change in dynamics of mortality evaluated by fixed basis change indexes indicates a substantial increase in this index, which in the medium- and long-term perspective was respectively 6.68 and 27.4. In contrast, in the same statistical analysis indexes of dynamic changes calculated both for surgical complications and re-interventions were much lower, up to 3 years not exceeding 2, up to 5 years not exceeding 3 (Table IV).

\section{Discussion}

Due to the low invasiveness, the endovascular technique has become the dominant treatment for AAA throughout the world. However, in Poland, it is just an alternative to open surgery for AAA patients $[1,3,5-7,16,17]$.

According to recommendations of the European Society of Vascular Surgery, EVAR is graded as an intermediate risk of intervention, defined as carrying a risk of cardiovascular death or myocardial infarction between $1 \%$ and $5 \%$ within 30 days. Open aortic repair is defined as a high risk intervention, as carrying a risk of cardiovascular death or myocardial infarction $5 \%$ or more within 30 days. In most patients with suitable anatomy and reasonable life expectancy EVAR should be considered as the preferred treatment modality [17]. In contrast, according to NICE guidelines EVAR should not be offered to patients with elective
AAA if open surgical repair is suitable. The decision regarding the choice of EVAR or open aortic repair also depends on the patient's preferences, so advantages and disadvantages of each method of surgical treatment of AAA should be presented [15].

The current study evaluated time-related changes in mortality, surgical complication and re-interventions of elective EVAR procedures among patients followed up for 5 years.

World studies including our results comparing cumulative rates of mortality, surgical complications and re-interventions in consecutive periods of time after planned EVAR are presented in Table V.

\section{Mortality}

World studies on AAA patients treated during elective admissions using the endovascular method show short-term mortality rates of 0 to $4.3 \%[1,5$, $7,13,15,18-30]$. The short-term mortality of $2.5 \%$ characterized the patients with elective endovascular treatment in the present study and is comparable to cited reports. Randomised controlled trials, OVER ACE, DREAM, and EVAR 1 demonstrated the short-term mortality rate for the use of the endovascular technique $0.5-1.7 \%[18,19,24,26]$. The highest short-term mortality rate $(4.3 \%)$ for endovascular treatment of AAA was found in a French report where the group of patients consisted of elderly people with numerous comorbidities, who were classified in the $3^{\text {rd }}$ and $4^{\text {th }}$ ASA categories for surgery-related risk [20].

In our study, medium- and long-term mortality rates for the patients were $14.2 \%$ and $28.9 \%$, which is similar to other reports. Two meta-analyses from the United Kingdom of the outcomes of endovascular treatment in elective AAA repair demonstrated the mortality rates for three periods of observation - up to 2 years, up to 4 years and at least 4 years after the surgery respectively $14.3 \%, 15.8 \%$ and 
Table V. Studies comparing cumulative rates of mortality, surgical complications and re-interventions in consecutive periods of time after elective endovascular aneurysm repair (EVAR)

\begin{tabular}{|c|c|c|c|c|c|c|c|c|c|}
\hline \multirow[t]{3}{*}{ Study } & \multicolumn{9}{|c|}{ Time of observation } \\
\hline & \multicolumn{3}{|c|}{ Up to 30 days after surgery } & \multicolumn{3}{|c|}{ Up to 3 years after surgery } & \multicolumn{3}{|c|}{ Up to 5 years after surgery } \\
\hline & $\begin{array}{c}\text { Mortality } \\
(\%)\end{array}$ & $\begin{array}{l}\text { Surgical } \\
\text { complica- } \\
\text { tions (\%) }\end{array}$ & $\begin{array}{l}\text { Re-inter- } \\
\text { ventions } \\
\quad(\%)\end{array}$ & $\begin{array}{c}\text { Mortality } \\
(\%)\end{array}$ & $\begin{array}{l}\text { Surgical } \\
\text { complica- } \\
\text { tions (\%) }\end{array}$ & $\begin{array}{l}\text { Re-inter- } \\
\text { ventions } \\
\quad(\%)\end{array}$ & $\begin{array}{c}\text { Mortality } \\
\text { (\%) }\end{array}$ & $\begin{array}{l}\text { Surgical } \\
\text { complica- } \\
\text { tions (\%) }\end{array}$ & $\begin{array}{l}\text { Re-inter- } \\
\text { ventions } \\
\quad(\%)\end{array}$ \\
\hline Own results & 2.5 & 7.6 & 4.2 & 14.2 & 12.6 & 7.5 & 28.9 & 17.5 & 11.4 \\
\hline Lee & 0 & - & 2 & - & - & - & 14 & - & 12 \\
\hline Jean-Baptiste & 4.3 & - & 4.3 & 15 & 10.9 & 4.3 & - & 23.2 & - \\
\hline DREAM & 1.2 & 19.9 & - & 10.3 & - & - & 31.1 & - & 29.6 \\
\hline EVAR 1 & 1.7 & - & 9.8 & - & - & - & 25 & - & 21 \\
\hline Sugimoto & - & - & - & - & - & - & 16.6 & 19.8 & 18.9 \\
\hline ACE & 1.3 & - & 5.3 & 17.6 & 17.4 & 16 & - & - & - \\
\hline Stather & 1.3 & - & - & 14.3 & - & - & 34.7 & - & - \\
\hline Sandford & 0 & 8 & 6.8 & - & - & - & - & - & 14 \\
\hline Muhs & 2.6 & - & - & 13 & 23.7 & 7.9 & - & - & - \\
\hline Verzini & 0.8 & 6.5 & 2 & - & - & - & 29.9 & - & 12.3 \\
\hline
\end{tabular}

$34.7-37.3 \%[13,25]$. For the AAA patients subjected to an elective endovascular procedure, the rate of medium-term mortality of the DREAM trial was $10.3 \%$, while in the long-term perspective the mortality rates were $25 \%$ in EVAR trial 1 and $31.1 \%$ in the DREAM trial [31-33].

\section{Surgical complications}

In the present study, the cumulative rates of surgical complications for short-, medium- and longterm perspectives were respectively $7.6 \%, 12.6 \%$ and $17.5 \%$. In separate time periods, up to 30 days after surgery, the most frequent complications were type I or II leaks (2.5\%) and thrombosis and stentgraft occlusion with acute ischaemia of a lower limb (2.5\%), in 30 days to 3 years and from 3 to 5 years after surgery the leak rates were $3.9 \%$ and $4.9 \%$, respectively.

Sandford et al. quoted the short-term surgical complications rate for the planned endovascular method as $8 \%$ and observed that type I or II leaks accounted for all cases of surgical complications for the observation period [23]. Ziaja et al. performed an analysis of surgical complications in a group of AAA patients subjected to elective endovascular treatment. Up to 30 days after surgery, type I or II leaks were observed in $17 \%$ of cases and thrombosis and occlusion of the stent-graft with acute ischaemia of a lower limb in $7.6 \%$ of patients [22]. In medium-term observation patients with AAA after elective implantation of the stent graft, Muhs et al. reported the occurrence of type I or II leaks in $20.6 \%$ of cases, respectively [28].

\section{Re-interventions}

The cumulative rates of re-interventions in short-, medium- and long-term perspectives in the current study were $4.2 \%, 7.5 \%$ and $11.4 \%$ respectively. According to analysed reports (Table IV), the re-intervention rate in patients with AAA after elective implantation of the stent graft for short-, medium- and long-term observation period ranges within respectively $2-9.8 \%, 4.3-16 \%$, and $12-29.6 \%[14,19,20$, 23, 24, 28-34].

\section{Limitations}

The study has limitations. The most important limitation is the lack of head to head comparison between EVAR and open surgery for the AAA patients. 
The long-term results were not compared in the context of the expected risk of cardiovascular death.

Systemic complications were not included in the analysis. Most deaths in the medium- and long-term perspective were due to old age and numerous comorbidities and were not associated with implantation of the stent graft.

Due to the low number, no patients with ruptured AAA were analysed, which could allow for a more thorough evaluation of the endovascular technique.

\section{Conclusions}

The present study analyses the treatment outcomes for 236 AAA patients subject to planned endovascular surgery between 2010 and 2015. The patients with AAA treated in the elective endovascular method demonstrated a low short-term mortality rate, which is no different from worldwide reports on the use of the endovascular technique $[1,5,7$, $13,15,18-30]$. Furthermore, in this study the observed and predicted short-term mortality rates according to the V-Possum scale were not significantly different ( $p=0.7993)$. In the investigated group of patients, the high mortality rate in the medium- and long-term perspective was linked with aging and numerous comorbidities, especially ischaemic heart disease. The clinical evaluation of the endovascular method in the study revealed relatively low values in the cumulative rates of surgical complications, and re-interventions within the separate investigated periods compared to other reports.

\section{Conflict of interest}

The authors declare no conflict of interest.

\section{References}

1. Budtz-Lilly J, Venermo M, Debus S, et al. Editor's Choice - As sessment of international outcomes of intact abdominal aortic aneurysm repair over 9 years. Eur J Vasc Endovasc Surg 2017; 54: 13-20.

2. Powell JT, Sweeting MJ, Ulug P, et al. Meta-analysis of individual-patient data from EVAR-1, DREAM, OVER and ACE trials comparing outcomes of endovascular or open repair for abdominal aortic aneurysm over 5 years. Br J Surg 2017; 104: 166-78.

3. Kulig P, Lewandowski K, Ziaja D, et al. Endovascular aneurysm repair or open aneurysm repair for the treatment of abdominal aortic aneurysm - the latest update. Pol Przegl Chir 2016; 88: 166-74.

4. Morisaki K, Matsumoto T, Matsubara Y, et al. Elective endovascular vs. open repair for abdominal aortic aneurysm in octogenarians. Vascular 2016; 24: 348-54
5. Thomas D, Anderson D, Hulten E, et al. Open versus endovascular repair of abdominal aortic aneurysm: incidence of cardiovascular events in 632 patients in a department of defense cohort over 6-year follow-up. Vascular 2015; 23: 234-9.

6. Mohan PP, Hamblin MH. Comparison of endovascular and open repair of ruptured abdominal aortic aneurysm in the United States in the past decade. Cardiovasc Intervent Radiol 2014; 37: 337-42.

7. Dua A, Kuy S, Lee CJ, et al. Epidemiology of aneurysm repair in the United States from 2000 to 2010. J Vasc Surg 2014; 59: 1512-7.

8. Kumar L, Cowled P, Boult M, et al. Type II endoleak after endovascular aneurysm repair: natural history and treatment outcomes. Ann Vasc Surg 2017; 44: 94-102.

9. England A, Mc Williams R. Endovascular aortic aneurysm repair (EVAR). Ulster Med J 2013; 82: 3-10.

10. Maleux G, Koolen M, Heye S. Complications after endovascular aneurysm repair. Semin Intervent Radiol 2009; 26: 3-9.

11. Moulakakis KG, Klonaris C, Kakisis J, et. al. Treatment of type II endoleak and aneurysm expansion after EVAR. Ann Vasc Surg 2017; 39: 56-66.

12. AbuRahma AF, Hass SM, AbuRahma ZT, et al. Management of immediate post-endovascular aortic aneurysm repair type la endoleaks and late outcomes. J Am Coll Surg 2017; 224: 740-8.

13. Paravastu SC, Jayarajasingam R, Cottam R, et al. Endovascular repair of abdominal aortic aneurysm. Cochrane Database Syst Rev 2014; 1: CD004178.

14. Lee K, Tang E, Dubois L, et al. Durability and survival are similar after elective endovascular and open repair of abdominal aortic aneurysms in younger patients. J Vasc Surg 2015; 61: 636-41.

15. NICE guideline DRAFT. Abdominal aortic aneurysm: diagnosis and management. 2018:3-36.

16. Kulig P, Lewandowski K, Banas B, et al. Short-term outcomes of endovascular repair of abdominal aortic aneurysm, including ruptured cases. Videosurgery Miniinv 2018; 13: 243-9.

17. Wanhainen A, Verzini F, Van Herzeele I, et al. European Society for Vascular Surgery (ESVS) 2019 clinical practice guidelines on the management of aorto-iliac artery aneurysms. Eur J Vasc Endovasc Surg 2019; 57: 8-93.

18. Lederle F, Freischlag J, Kyriades T, et al. Outcomes following endovascular vs open repair of abdominal aortic aneurysm: a randomized trial. JAMA 2009; 302: 1535-42.

19. Greenhalgh RM, Brown LC, Kwong GP, et al. Comparison of endovascular aneurysm repair with open repair in patients with abdominal aortic aneurysm (EVAR trial 1), 30-day operative mortality results: randomised controlled trial. Lancet 2004; 364: 843-8.

20. Jean-Baptiste E, Hassen-Khodja R, Bouillanne PJ, et al. Endovascular repair of infrarenal abdominal aortic aneurysms in high-risk-surgical patients. Eur J Vasc Endovasc Surg 2007; 34: 145-51.

21. Gnus J, Ferenc S, Dziewiszek M, et al. Comparison of endovascular aneurysm repair with open repair in patients with abdominal aortic aneurysm in our own material in years 20022011. Adv Clin Exp Med 2015; 24: 475-9.

22. Ziaja K, Kuczmik W, Ziaja D, et al. Endovascular treatment of infrarenal aortic aneurysm - three years of experience. Chirurg Pol 2003; 5: 129-43. 
23. Sandford RM, Choke E, Bown MJ, et al. What is the best option for elective repair of an abdominal aortic aneurysm in a young fit patient? EurJ Vasc and Endovasc Surg 2014; 47: 13-8.

24. Becquemin JP, Pillet JC, Lescalie F, et al. A randomized controlled trial of endovascular aneurysm repair versus open surgery for abdominal aortic aneurysms in - to moderate-risk patients. J Vasc Surg 2011; 53: 1167-73.

25. Stather PW, Sidloff D, Dattani N, et al. Systematic review and meta-analysis of the early and late outcomes of open and endovascular repair of abdominal aortic aneurysm. Br J Surg 2013; 100: 863-72.

26. Prinssen M, Verhoeven E, Buth J, et al. A randomized trial comparing conventional and endovascular repair of abdominal aortic aneurysms. N Engl J Med 2004; 351: 1607-18.

27. Mazzaccaro D, Nano G, Settembrini AM, et al. Open and endovascular elective treatment of abdominal aortic aneurysms: a real-world experience. Surg Today 2017; 47: 1347-55.

28. Muhs B, Verhoeven E, Zeebregts C, et al. Mid-term results of endovascular aneurysm repair with branched and fenestrated endografts. J Vasc Surg 2006; 44: 9-15.

29. Qadura M, Pervaiz F, Harlock JA, et. al. Mortality and reintervention following elective abdominal aortic aneurysm repair. J Vasc Surg 2013; 57: 1676-83.

30. Verzini F, Romano L, Parlani G, et al. Fourteen-year outcomes of abdominal aortic endovascular repair with the Zenith stent graft. J Vasc Surg 2017; 65: 318-29.

31. Blankensteijn JD, De Jong SE, Prinssen M, et al. Two-year outcomes after conventional or endovascular repair of abdominal aortic aneurysms. N Engl J Med 2005; 352: 2398-405.

32. De Bruin JL, Baas AF, Buth J, et al. Long-term outcome of open or endovascular repair of abdominal aortic aneurysm. N Engl J Med 2010; 362: 1881-9.

33. Patel R, Sweeting MJ, Powell JT, et al. Endovascular versus open repair of abdominal aortic aneurysm in 15-years follow up of the UK endovascular aneurysm repair trial 1 (EVAR trial 1) a randomized controlled trial. Lancet 2016; 388: 2366-74

34. Sugimoto $M$, Koyama A, Niimi K, et al. Long-term comparison of endovascular and open repair of abdominal aortic aneurysms: retrospective analysis of matched cohorts with propensity score. Ann Vasc Surg 2017; 43: 96-103.

Received: 23.12.2019, accepted: 23.02.2020 